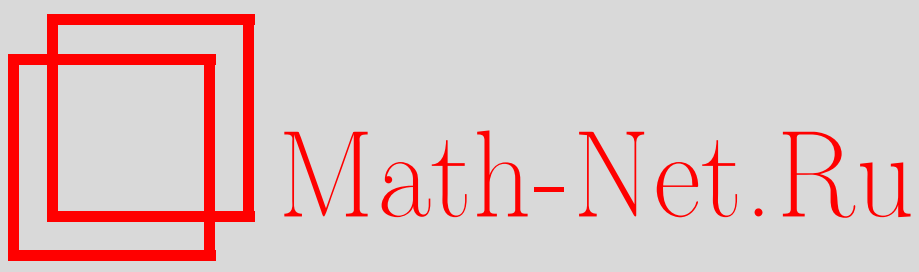

V. N. Zhelyabin, A. S. Zakharov, The superalgebras of jordan brackets defined by the n-dimensional sphere, Sibirsk. Mat. Zh., 2020, Volume 61, Number 4, 803-822

DOI: https://doi.org/10.33048/smzh.2020.61.407

Use of the all-Russian mathematical portal Math-Net.Ru implies that you have read and agreed to these terms of use http://www . mathnet.ru/eng/agreement

Download details:

IP : 34.229 .108 .108

April 26, 2023, 16:59:30

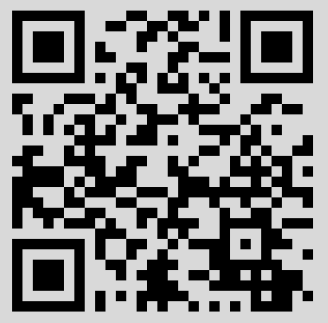


Сибирский математический журнал Июль-август, 2020. Том 61, № 4

УДК 512.554

\title{
СУПЕРАЛГЕБРЫ ЙОРДАНОВЫХ СКОБОК, ОПРЕДЕЛЕННЫЕ $n$-МЕРНОЙ СФЕРОЙ
}

\section{В. Н. Желябин, А. С. Захаров}

\begin{abstract}
Аннотация. Изучаются обобщенные скобки Лейбница, заданные на координатной алгебре $n$-мерной сферы. В случае одномерной сферы показано, что любая такая скобка является скобкой векторного типа. Любая йорданова скобка, заданная на координатной алгебре двумерной сферы, является обобщенной скобкой Пуассона. На координатной алгебре сферы нечетной размерности можно задать йорданову скобку, дубль Кантора которой является простой йордановой супералгеброй. С использованием таких супералгебр построены примеры простых абелевых йордановых супералгебр, нечетная часть которых является конечнопорожденным проективным модулем ранга 1 с любым числом порождающих. Аналогичный результат имеет место для декартова произведения сферы четной размерности и аффинной прямой. В частности, для случая двумерной сферы полученная йорданова супералгебра исключительная. Построенные супералгебры являются новыми примерами простых йордановых супералгебр.
\end{abstract}

DOI 10.33048/smzh.2020.61.407

Ключевые слова: ассоциативная коммутативная супералгебра, йорданова супералгебра, дифференциальная алгебра, алгебра Грассмана, супералгебра билинейной формы, алгебра полиномов, дифференцирование, йорданова скобка, скобка векторного типа, скобка Пуассона, проективный модуль, аффинное пространство, сфера.

Супералгебры йордановых скобок имеют большое значение в исследовании структуры йордановых алгебр и супералгебр. Примеры супералгебр йордановых скобок можно получить, используя процесс удвоения Кантора, из ассоциативной коммутативной супералгебры, на которой задана йорданова скобка (см. [1-3]). Основные свойства супералгебр йордановых скобок такие, как, например, специальность, изучались в работах [1-11].В $[12,13]$ показано, что коммутатор относительно операции умножения алгебры Новикова на ассоциативной коммутативной части алгебры Новикова - Пуассона определяет йорданову скобку. В [14] показано, что йорданова супералгебра, построенная по этой скобке, специальная. Значительную роль (см. $[5,6,15])$ супералгебры йордановых скобок векторного типа сыграли в изучении первичных вырожденных йордановых алгебр.

Заметим, что если йорданова скобка задана на ассоциативно коммутативной алгебре, то четная часть полученной йордановой супералгебры ассоциативна, а нечетная является ассоциативным модулем над четной частью. Такие супералгебры, следуя [16], будем называть абелевыми.

Простые йордановы абелевы супералгебры близки по свойствам к супералгебрам йордановых скобок. Простые йордановы супералгебры с ассоциативной

В. Н. Желябин выполнял исследования в рамках государственного задания ИМ СО РАН, проект 0314-2019-0001.

(c) 2020 Желябин В. Н., Захаров А. С. 
четной частью изучались в $[17-23]$. В $[17,18]$ описаны простые унитальные специальные абелевы йордановы супералгебры, неизоморфные супералгебре невырожденной билинейной формы. Как оказалось, такие супералгебры являются супералгебрами векторного типа относительно нескольких дифференцирований, и их нечетная часть - конечнопорожденный проективный модуль ранга 1. Более того, каждая такая супералгебра вкладывается в простую супералгебру векторного типа, построенную с помощью одного дифференцирования. В [1821] были построены примеры простых йордановых супералгебр векторного типа относительно двух дифференцирований. Более точно, нечетная часть таких супералгебр является проективным модулем ранга 1 и порождается как модуль не менее чем двумя элементами. Примеры первичных йордановых абелевых супералгебр векторного типа, у которых нечетная часть является конечнопорожденным проективным модулем ранга 1 с произвольным числом порождающих, построены в [24].

В [23] показано, что простая, не обязательно специальная, йорданова абелева супералгебра вкладывается в простую супералгебру йордановой скобки. Более того, нечетная часть такой супералгебры как модуль над четной частью является конечнопорожденным проективным модулем ранга 1.

Следует отметить работу [25], которая послужила началом изучения простых йордановых супералгебр с ассоциативной четной частью. Одним из методов исследования настоящей работы является изучение действия подалгебры алгебры Ли векторных полей $n$-мерной сферы на алгебре регулярных функций $n$-мерной сферы. В этой связи отметим работы [26-28].

Работа состоит из трех параграфов. В $\S 1$ изучаются условия простоты супералгебры, построенной процессом удвоения Кантора с помощью обобщенной скобки Лейбница. Найдены эквивалентные условия простоты супералгебры и некоторой ее подалгебры для скобки, заданной на ассоциативной коммутативной $Z_{2}$-градуированной алгебре. Также приведен критерий простоты супералгебры в терминах дифференциальной простоты алгебры относительно некоторого множества дифференцирований. В $\S 2$ изучаются свойства обобщенной скобки Лейбница, заданной на алгебре регулярных функций $n$-мерной сферы. В 33 строятся новые примеры простых унитальных абелевых йордановых супералгебр, не изоморфных супералгебре йордановой скобки.

\section{$\S 1$. Обобщенные скобки Лейбница и дубль Кантора}

Пусть $F-$ поле характеристики не 2 . Алгебра $A=A_{0}+A_{1}-Z_{2}$-градуированная алгебра, если $A_{0} \cap A_{1}=0$ и $A_{i} A_{j}=A_{i+j \bmod 2 .}$. Алгебру $A$ будем называть супералгеброй. Пространство $A_{0}\left(A_{1}\right)$ называется четной (нечетной) частъю $Z_{2}$-градуированной алгебры $A$. Элементы множества $A_{0} \cup A_{1}$ называются однородными. Выражение $|x|$, где $x \in A_{0} \cup A_{1}$, означает индекс четности однородного элемента $x$ :

$$
|x|=\left\{\begin{array}{lll}
0, & \text { если } x \in A_{0} & (x \text { четный }), \\
1, & \text { если } x \in A_{1} & (x \text { нечетный }) .
\end{array}\right.
$$

Пусть $G$ - алгебра Грассмана с единицей 1 над $F$, т. е. ассоциативная алгебра, заданная образующими $1, e_{1}, e_{2}, \ldots$ и определяющими соотношениями

$$
e_{i}^{2}=0, \quad e_{i} e_{j}=-e_{j} e_{i} .
$$

Произведения $1, e_{i_{1}} \ldots e_{i_{k}}$, где $i_{1}<i_{2}<\cdots<i_{k}$, образуют базис алгебры $G$. Пусть $G_{0}, G_{1}$ - векторные подпространства, порожденные соответственно про- 
изведениями четной и нечетной длины. Тогда $G=G_{0}+G_{1}-Z_{2}$-градуированная алгебра.

Пусть $A=A_{0}+A_{1}-Z_{2}$-градуированная алгебра. Тогда $G(A)=G_{0} \otimes A_{0}+$ $G_{1} \otimes A_{1}$ является подалгеброй в алгебре $G \otimes A$ (тензорное произведение над полем $F$ ) и называется грассмановой оболочкой алгебры $A$.

Ассоциативная супералгебра $A=A_{0}+A_{1}$ называется ассоциативной коммутативной супералгеброй, когда ее грассманова оболочка $G(A)$ - ассоциативная коммутативная алгебра. Тогда в супералгебре $A$ для однородных элементов выполняется тождество

$$
a b=(-1)^{|a||b|} b a .
$$

Пусть $\Gamma=\Gamma_{0}+\Gamma_{1}-$ ассоциативная коммутативная супералгебра над полем $F$ и $\{\}:, \Gamma \times \Gamma \mapsto \Gamma-$ суперкососимметрическое билинейное отображение супералгебры $\Gamma$, которое будем называть скобкой. По супералгебре $\Gamma$ и скобке $\{$,$\} можно построить супералгебру J(\Gamma,\{\}$,$) . Рассмотрим J(\Gamma,\{\})=,\Gamma \oplus \Gamma \xi$, прямую сумму векторных пространств, где $\Gamma \xi-$ изоморфная копия пространства $\Gamma$. Операция умножения $(\cdot)$ на $J(\Gamma,\{\}$,$) определяется формулами$

$$
a \cdot b=a b, \quad a \cdot b \xi=(a b) \xi, \quad a \xi \cdot b=(-1)^{|b|}(a b) \xi, \quad a \xi \cdot b \xi=(-1)^{|b|}\{a, b\},
$$

где $a, b \in \Gamma_{0} \cup \Gamma_{1}$ и $a b-$ произведение элементов $a, b$ в $\Gamma$. Положим $A_{0}=\Gamma_{0}+\Gamma_{1} \xi$ и $A_{1}=\Gamma_{0} \xi+\Gamma_{1}$. Тогда $J(\Gamma,\{\})=,A_{0}+A_{1}$ является супералгеброй и называется дублем Кантора.

Пусть $D$ - четное дифференцирование супералгебры $\Gamma$, т. е. $D\left(\Gamma_{i}\right) \subseteq \Gamma_{i}$, $i=0,1$. Тогда скобка $\{$,$\} называется обобщенной скобкой Лейбница или D$ скобкой Лейбница, если для $a, b, c \in \Gamma_{0} \cup \Gamma_{1}$ выполняется равенство

$$
\{a, b c\}=\{a, b\} c+(-1)^{|a||b|} b\{a, c\}-D(a) b c .
$$

Если $D=0$, то скобка $\{$,$\} называется скобкой Лейбница.$

Заметим, что если $\{$,$\} - обобщенная скобка Лейбница, то$

$$
\langle a, b\rangle=\{a, b\}-D(a) b+a D(b)
$$

является скобкой Лейбница.

Пусть $\Gamma-$ унитальная супералгебра. Подставляя $b=c=1$ в (1), получим $D(a)=\{a, 1\}$.

Для обобщенной скобки Лейбница $\{$,$\} определим якобиан$

$$
J(a, b, c)=\{a,\{b, c\}\}+(-1)^{|a||b|+|a||c|}\{b,\{c, a\}\}+(-1)^{|a||c|+|b||c|}\{c,\{a, b\}\}
$$

элементов $a, b, c$ и

$$
S(a, b, c)=\{a, b\} D(c)+(-1)^{|a||b|+|a||c|}\{b, c\} D(a)+(-1)^{|a||c|+|b||c|}\{c, a\} D(b) .
$$

Если $\Gamma$ - унитальная супералгебра, то $S(1, a, b)=0$ для любых $a, b \in \Gamma$.

Лемма 1. Пусть $\Gamma=\Gamma_{0}+\Gamma_{1}-$ ассоциативная коммутативная супералгебра и $\{$,$\} - обобщенная скобка Лейбница на Г. Тогда для однородных элементов$ имеют место следующие тождества:

$$
\begin{gathered}
S(a b, u, v)=a S(b, u, v)+(-1)^{|a||b|} b S(a, u, v), \\
J(a b, u, v)=a J(b, u, v)+(-1)^{|a||b|} b J(a, u, v)+a b(D(\{u, v\})-\{D(u), v\}-\{u, D(v)\}) .
\end{gathered}
$$


Если Г - унитальная супералгебра, то

$$
J(a b, u, v)=a J(b, u, v)+(-1)^{|a||b|} b J(a, u, v)-a b J(1, u, v) .
$$

ДокАзАТЕльство. Указанные тождества достаточно доказать для ассоциативной коммутативной алгебры Г. Покажем (3). Пусть $a, b, u, v \in \Gamma$. Тогда

$$
\begin{gathered}
S(a b, u, v)=\{a b, u\} D(v)+\{u, v\} D(a b)+\{v, a b\} D(u) \\
=(a\{b, u\}+\{a, u\} b+a b D(u)) D(v)+\{u, v\} D(a b)+(\{v, a\} b+a\{v, b\}-D(v) a b) D(u) \\
=a(\{b, u\} D(v)+\{u, v\} D(b)+\{v, b\} D(u)) \\
+b(\{a, u\} D(v)+\{u, v\} D(a)+\{v, a\} D(u))=a S(b, u, v)+b S(a, u, v) .
\end{gathered}
$$

Аналогично доказывается тождество (4).

Пусть Г - унитальная супералгебра. Тогда

$$
J(1, u, v)=-(D(\{u, v\})-\{D(u), v\}-\{u, D(v)\}) .
$$

Отсюда следует (5).

Лемма 2. Пусть $\Gamma=\Gamma_{0}+\Gamma_{1}-$ унитальная ассоциативная коммутативная супералгебра и $\{$,$\} - обобщенная скобка Лейбница на Г. Определим скобку$

$$
\langle a, b\rangle=\{a, b\}-D(a) b+D(b) a .
$$

Тогда

$$
\begin{aligned}
J(a, b, c)_{\langle,\rangle}=J(a, b, c)+ & S(a, b, c)-a J(1, b, c) \\
& -(-1)^{|a|(|b|+|c|)} b J(1, c, a)-(-1)^{|c|(|a|+|b|)} c J(1, a, b),
\end{aligned}
$$

где $J(a, b, c)_{\langle,\rangle}$- якобиан скобки $\langle$,$\rangle .$

ДокАЗАТЕЛЬСтво. По определению скобки $\langle$,$\rangle имеем$

$$
\begin{gathered}
\langle a,\langle b, c\rangle\rangle=\{a,\langle b, c\rangle\}-D(a)\langle b, c\rangle+a D(\langle b, c\rangle)=\{a,\{b, c\}\}+\{a, b\} D(c)+a D(\{b, c\}) \\
-\{a, D(b)\} c+(-1)^{|a||b|} b\{a, D(c)\}-D(a)\{b, c\}-(-1)^{|a||b|} D(b)\{a, c\} \\
-2 D(a) b D(c)+2 D(a) D(b) c-a D^{2}(b) c+a b D^{2}(c) .
\end{gathered}
$$

Отсюда непосредственным вычислением получаем искомый результат.

Пусть $\Lambda=\Lambda_{0}+\Lambda_{1}-Z_{2}$-градуированная ассоциативная коммутативная алгебра. Положим $\Gamma_{0}=\Lambda, \Gamma_{1}=0$ и $\Gamma=\Gamma_{0}+\Gamma_{1}=\Lambda$. Предположим, что на $\Lambda$ задана обобщенная скобка Лейбница, согласованная с $Z_{2}$-градуировкой алгебры $\Lambda$. Тогда $J\left(\Lambda_{0}, \Lambda_{1},\{\},\right)=\Lambda_{0}+\Lambda_{1} \xi-$ подсупералгебра супералгебры $J(\Lambda,\{\})=,J(\Gamma,\{\}$,$) .$

Теорема 1. Пусть $\Lambda-Z_{2}$-градуированная унитальная ассоциативная коммутативная алгебра без делителей нуля и $\Lambda_{0}=\Lambda_{1} \Lambda_{1}$. Тогда супералгебра $J(\Lambda,\{\}$,$) проста в том и только в том случае, когда подсупералгебра J\left(\Lambda_{0}, \Lambda_{1}\right.$, $\{\}$,$) проста.$

ДокАЗАТЕЛЬСтво. Пусть подсупералгебра $J\left(\Lambda_{0}, \Lambda_{1},\{\},\right)$ проста. Предположим, что $I-$ идеал супералгебры $J(\Lambda,\{\}$,$) . Тогда I=K+L \xi$. Поскольку $I$ - идеал, то $K, L$ - идеалы алгебры $\Lambda$. Аналогично

$$
K \xi \subseteq(K \Lambda) \xi \subseteq K \cdot \Lambda \xi \subseteq L \xi, \quad\{K, \Lambda\} \subseteq K \xi \cdot \Lambda \xi \subseteq L \xi \cdot \Lambda \xi \subseteq K .
$$


Супералгебры йордановых скобок, определенные $n$-мерной сферой 807

Пусть $K_{0}=K \cap \Lambda_{0}$. Тогда $K_{0}$ - идеал алгебры $\Lambda_{0}$ и $\left\{K_{0} \Lambda_{1}, \Lambda_{1}\right\} \subseteq K \cap \Lambda_{0}=$ $K_{0}$. Положим $R=K_{0}+\left(K_{0} \Lambda_{1}\right) \xi$. Тогда $R \cdot \Lambda_{0} \subseteq R$ и

$$
R \cdot \Lambda_{1} \xi \subseteq K_{0} \cdot \Lambda_{1} \xi+\left\{K_{0} \Lambda_{1}, \Lambda_{1}\right\} \subseteq R .
$$

Следовательно, $R$ - идеал супералгебры $J\left(\Lambda_{0}, \Lambda_{1},\{\},\right)$. Тогда либо $1 \in K_{0}$, либо $K_{0}=0$. Если $1 \in K_{0}$, то $1 \in K$ и $I=J(\Lambda,\{\}$,$) .$

Пусть $K_{0}=0$ и $a_{0}+a_{1} \in K$, где $a_{0} \in \Lambda_{0}, a_{1} \in \Lambda_{1}$. Тогда

$$
\left(a_{0}+a_{1}\right)\left(a_{0}-a_{1}\right)=a_{0}^{2}-a_{1}^{2} \in K \cap \Lambda_{0}=K_{0}=0 .
$$

Поскольку алгебра $\Lambda$ не содержит делителей нуля, то $a_{0}+a_{1}=0$ и $K=0$.

Таким образом, $J(\Lambda,\{\}$,$) - простая супералгебра.$

Пусть $J(\Lambda,\{\}$,$) - простая супералгебра. Предположим, что I$ - идеал супералгебры $J\left(\Lambda_{0}, \Lambda_{1},\{\},\right)$. Тогда $I=K_{0}+K_{1} \xi$, где $K_{0}$ - идеал алгебры $\Lambda_{0}$, а $K_{1}$ - подмодуль $\Lambda_{0}$-модуля $\Lambda_{1}$. Также получаем

$$
\left\{K_{0} \Lambda_{1}, \Lambda_{1}\right\} \subseteq\left(K_{0} \cdot \Lambda_{1} \xi\right) \cdot \Lambda_{1} \xi \subseteq K_{1} \xi \cdot \Lambda_{1} \xi \subseteq K_{0} .
$$

Поскольку $\{$,$\} - обобщенная скобка Лейбница и \Lambda_{0}=\Lambda_{1} \Lambda_{1}$, то

$$
\begin{aligned}
\left\{K_{0}, \Lambda_{1}\right\} \subseteq\left\{\Lambda_{1}, K_{0}\right\} \subseteq\left\{\Lambda_{1}, K_{0} \Lambda_{1} \Lambda_{1}\right\} & \\
& \subseteq\left\{K_{0} \Lambda_{1}, \Lambda_{1}\right\} \Lambda_{1}+K_{0} \Lambda_{1}\left\{\Lambda_{1}, \Lambda_{1}\right\}+D\left(\Lambda_{1}\right) K_{0} \Lambda_{1} \Lambda_{1} \subseteq K_{0} \Lambda_{1} .
\end{aligned}
$$

Так как $1 \in \Lambda_{1} \Lambda_{1}$, то $1=\sum_{i} a_{i} b_{i}$, где $a_{i}, b_{i} \in \Lambda_{1}$. Пусть $r \in K_{0}$. Тогда

$$
D(r)=\{r, 1\}=\left\{r, \sum_{i} a_{i} b_{i}\right\}=\sum_{i} a_{i}\left\{r, b_{i}\right\}+\sum_{i}\left\{r, a_{i}\right\} b_{i}-\sum_{i} D(r) a_{i} b_{i} .
$$

Поскольку

$$
\sum_{i} a_{i}\left\{r, b_{i}\right\}+\sum_{i}\left\{r, a_{i}\right\} b_{i} \in\left\{K_{0}, \Lambda_{1}\right\} \Lambda_{1} \subseteq K_{0} \Lambda_{1} \Lambda_{1} \subseteq K_{0} \Lambda_{0} \subseteq K_{0},
$$

то $2 D(r) \in K_{0}$. Отсюда получаем

$$
\left\{K_{0}, \Lambda_{0}\right\} \subseteq\left\{K_{0}, \Lambda_{1} \Lambda_{1}\right\} \subseteq\left\{K_{0}, \Lambda_{1}\right\} \Lambda_{1}+D\left(K_{0}\right) \Lambda_{1} \Lambda_{1} \subset K_{0} .
$$

Аналогично $\left\{K_{0} \Lambda_{1}, \Lambda_{0}\right\} \subseteq K_{0} \Lambda_{1}$.

Пусть $K=K_{0}+K_{0} \Lambda_{1}$. Тогда $K-$ идеал алгебры $\Lambda$ и

$$
\{K, \Lambda\} \subseteq\left\{K_{0}, \Lambda\right\}+\left\{K_{0} \Lambda_{1}, \Lambda_{0}\right\}+\left\{K_{0} \Lambda_{1}, \Lambda_{1}\right\} \subseteq K_{0}+K_{0} \Lambda_{1}=K .
$$

Поэтому $K+K \xi-$ идеал супералгебры $J(\Lambda,\{\}$,$) . Следовательно, либо 1 \in K_{0}$, либо $K_{0}=0$. Если $1 \in K_{0}$, то $I=J\left(\Lambda_{0}, \Lambda_{1},\{\},\right)$.

Пусть $K_{0}=0$. Тогда $I=K_{1} \xi$. Поэтому

$$
\left\{K_{1}, \Lambda_{1}\right\} \subseteq K_{1} \xi \cdot \Lambda_{1} \xi \subseteq K_{0}=0 .
$$

Следовательно, $K_{1} \Lambda_{1}+K_{1} \xi-$ идеал супералгебры $J\left(\Lambda_{0}, \Lambda_{1},\{\},\right)$. В силу доказанного выше либо $1 \in K_{1} \Lambda_{1}$, либо $K_{1} \Lambda_{1}=0$.

Если $1 \in K_{1} \Lambda_{1}$, то $\Lambda_{1}=K_{1}$ и $\left\{\Lambda_{1}, \Lambda_{1}\right\}=0$. Пусть $a \in \Lambda_{1}$ и $1=\sum_{i} a_{i} b_{i}$, где $a_{i}, b_{i} \in \Lambda_{1}$. Тогда

$D(a)=\{a, 1\}=\left\{a, \sum_{i} a_{i} b_{i}\right\}=\sum_{i} a_{i}\left\{a, b_{i}\right\}+\sum_{i}\left\{a, a_{i}\right\} b_{i}-\sum_{i} D(a) a_{i} b_{i}=-D(a)$. 
Поэтому $2 D(a)=0$. Отсюда получаем

$$
\left\{\Lambda_{1}, \Lambda_{0}\right\} \subseteq\left\{\Lambda_{1}, \Lambda_{1} \cdot \Lambda_{1}\right\} \subseteq\left\{\Lambda_{1}, \Lambda_{1}\right\} \Lambda_{1}+D\left(\Lambda_{1}\right) \Lambda_{1} \cdot \Lambda_{1}=0 .
$$

Аналогично $\left\{\Lambda_{0}, \Lambda_{0}\right\}=0$. Поэтому $\{\Lambda, \Lambda\}=0$ и $\Lambda \xi-$ идеал супералгебры $J(\Lambda,\{\}$,$) . Следовательно, 1 \notin K_{1} \Lambda_{1}$. Тогда $K_{1} \Lambda_{1}=0$ и $K_{1}=0$, так как $\Lambda_{1} \Lambda_{1}=\Lambda_{0}$. Поэтому $I=0$.

Таким образом, $J\left(\Lambda_{0}, \Lambda_{1},\{\},\right)$ - простая супералгебра.

Пусть $\Lambda$ - унитальная ассоциативная коммутативная алгебра, на которой задана обобщенная скобка Лейбница $\{$,$\} . Для элементов u, v \in \Lambda$ определим отображение $D_{u, v}: \Lambda \mapsto \Lambda$, полагая

$$
D_{u, v}(a)=\{a u, v\}-a\{u, v\}
$$

Лемма 3. Отображение $D_{u, v}$ - дифференцирование алгебры $\Lambda$. Если супералгебра $J(\Lambda,\{\}$,$) проста, то алгебра \Lambda$ дифференциально проста относительно дифференцирований из $\mathscr{D}=\left\{D_{u, v} \mid u, v \in \Lambda\right\}$. Обратно, если алгебра $\Lambda$ дифференциально проста относительно дифференцирований из $\mathscr{D}$, то либо $\{\Lambda, \Lambda\}=0$, либо $J(\Lambda,\{\})-$, простая супералгебра.

ДокАЗАТЕЛЬСтво. Пусть $a, b \in \Lambda, u, v \in \Lambda$. В силу (1)

$$
\begin{aligned}
D_{u, v}(a b)=\{a b u, v\}- & a b\{u, v\}=a\{b u, v\}+\{a, v\} b u+D(v) a b u-a b\{u, v\} \\
& =a D_{u, v}(b)+b\{a u, v\}-b a\{u, v\}=D_{u, v}(a) b+a D_{u, v}(b) .
\end{aligned}
$$

Следовательно, $D_{u, v}$ - дифференцирование алгебры $\Lambda$.

Пусть супералгебра $J(\Lambda,\{\}$,$) проста. Предположим, что I$ - идеал алгебры $\Lambda$, инвариантный относительно дифференцирований из $\mathscr{D}$. Тогда для любых $u, v \in \Lambda$ получаем

$$
\{I u, v\} \subseteq D_{u, v}(I)+I\{u, v\} \subseteq I .
$$

Поэтому $\{I \Lambda, \Lambda\} \subseteq I$. Следовательно, $\{I, \Lambda\} \subseteq I$.

Пусть $K=I+I \xi$. Тогда $K \cdot \Lambda \subseteq K$ и $K \cdot \Lambda \xi \subseteq K+\{I, \Lambda\} \subseteq K$. Следовательно, $K$ - идеал супералгебры $J(\Lambda,\{\}$,$) . Поскольку супералгебра J(\Lambda,\{\}$,$) проста,$ либо $K=0$, либо $K=J(\Lambda,\{\}$,$) . Отсюда получаем, что либо I=0$, либо $I=\Lambda$.

Пусть алгебра $\Lambda$ дифференциально проста относительно дифференцирований из $\mathscr{D}$. Предположим, что $I$ - идеал супералгебры $J(\Lambda,\{\}$,$) . Тогда$ $I=K+L \xi$, где $K, L-$ идеалы алгебры $\Lambda$. Кроме того,

$$
\{K \Lambda, \Lambda\} \subseteq(K \cdot \Lambda \xi) \cdot \Lambda \xi \subseteq L \xi \cdot \Lambda \xi \subseteq K .
$$

Следовательно, $K$ - идеал алгебры $\Lambda$, инвариантный относительно дифференцирований из $\mathscr{D}$. Тогда либо $1 \in K$, либо $K=0$. Можно считать, что $K=0$. Поэтому $I=L \xi$ и

$$
\{L, \Lambda\} \subseteq L \xi \cdot \Lambda \xi \subseteq K=0 .
$$

Отсюда получаем, что $L-$ идеал алгебры $\Lambda$, инвариантный относительно дифференцирований из $\mathscr{D}$. Тогда либо $1 \in L$, либо $L=0$. Если $1 \in L$, то $L=\Lambda$. Следовательно, $\{\Lambda, \Lambda\}=0$. Поэтому если $\{\Lambda, \Lambda\} \neq 0$, то $1 \notin L$. Отсюда следует $L=0$ и $I=0$.

Лемма доказана. 
Лемма 4. Пусть $\Lambda=\Lambda_{0}+\Lambda_{1}-$ унитальная ассоциативная коммутативная $Z_{2}$-градуированная алгебра, $\mathscr{D}-$ множество четных дифференцирований алгебры $\Lambda$. Предположим, что $\Lambda$ дифференциально проста относительно дифференцирований из $\mathscr{D}$ как $Z_{2}$-градуированная алгебра. Тогда либо $\Lambda_{1}=0$, либо $\Lambda_{0}=\Lambda_{1} \Lambda_{1}$. Кроме того, либо алгебра $\Lambda$ дифференциально проста относительно дифференцирований из $\mathscr{D}$, либо $\Lambda=\Lambda_{0}+s \Lambda_{0}$, где $s \in \Lambda_{1}, s^{2}=1, D(s)=0$ для любого $D \in \mathscr{D}$.

ДокАЗАТЕЛЬСТво. Не ограничивая общности, можно считать, что $\mathscr{D}=$ $\{D\}$. Заметим, что $\Lambda_{1} \Lambda_{1}+\Lambda_{1}-Z_{2}$-градуированный идеал $\Lambda$, инвариантный относительно $D$. Если $\Lambda_{1} \Lambda_{1}=0$, то $\Lambda_{1}-Z_{2}$-градуированный идеал алгебры $\Lambda$, инвариантный относительно $D$. Поэтому либо $\Lambda_{1}=0$, либо $\Lambda_{0}=\Lambda_{1} \Lambda_{1}$.

Пусть $I-$ ненулевой отличный от $\Lambda$ идеал алгебры $\Lambda$, инвариантный относительно $D$. Можно считать, что $\Lambda_{1} \neq 0$. Положим $I_{0}=I \cap \Lambda_{0}$. Тогда $I_{0}+I_{0} \Lambda_{1}-$ $Z_{2}$-градуированный идеал алгебры $\Lambda$, инвариантный относительно $D$. Поэтому либо $1 \in I_{0}$, либо $I_{0}=0$. Следовательно, можно считать, что $I_{0}=I \cap \Lambda_{0}=0$. Положим $I_{1}=I \cap \Lambda_{1}$. Тогда $I_{1} \Lambda_{1}+I_{1}-Z_{2}$-градуированный идеал алгебры $\Lambda$, инвариантный относительно $D$. Поэтому либо $I_{1}=\Lambda_{1}$, либо $I_{1}=0$. Поскольку $I_{1} \Lambda_{1}+I_{1} \subseteq I$, можно считать, что $I_{1}=I \cap \Lambda_{1}=0$.

Пусть

$$
I_{0}=\left\{a_{0} \in \Lambda_{0} \mid \exists a_{1} \in \Lambda_{1}, a_{0}+a_{1} \in I\right\} .
$$

Тогда $I_{0}-$ идеал $\Lambda_{0}$ и $D\left(I_{0}\right) \subseteq I_{0}$. Следовательно, $I_{0}+I_{0} \Lambda_{1}-Z_{2}$-градуированный идеал алгебры $\Lambda$, инвариантный относительно $D$. Поэтому либо $I_{0}=0$, либо $I_{0}=\Lambda_{0}$. Если $I_{0}=0$, то $I \subseteq I \cap \Lambda_{1}=0$.

Стало быть, $I_{0}=\Lambda_{0}$. Тогда $1+s \in I$ для некоторого $s \in \Lambda_{1}$. Отсюда

$$
(1+s)(1-s)=1-s^{2} \in I \cap \Lambda_{0}=0 .
$$

Следовательно, $s^{2}=1, \Lambda_{1}=s \Lambda_{0}$. Кроме того,

$$
D(s)=D(1+s) \in I \cap \Lambda_{1}=0 .
$$

Лемма доказана.

Лемма 5. Пусть $\Lambda=\Lambda_{0}+\Lambda_{1}$ - унитальная ассоциативная коммутативная $Z_{2}$-градуированная алгебра и $\Lambda_{0}=\Lambda_{1} \Lambda_{1}$. Тогда $\Lambda_{1}-$ конечнопорожденный проективный $\Lambda_{0}$-модуль ранга 1.

ДоказАтЕЛЬство. Пусть $\Lambda_{0}=\Lambda_{1} \Lambda_{1}$. Тогда $1=\sum_{i}=x_{i} y_{i}$, где $x_{i}, y_{i} \in \Lambda_{1}$. Следовательно,

$$
\Lambda_{1}=\Lambda_{0} x_{1}+\cdots+\Lambda_{0} x_{n},
$$

т. е. $\Lambda_{1}$ - конечнопорожденный $\Lambda_{0}$-модуль. Рассмотрим $\Lambda_{0}$-модуль $\Lambda_{1} \otimes_{\Lambda_{0}} \Lambda_{1}$. Тогда элемент $x_{1} \otimes y_{1}+\cdots+x_{n} \otimes y_{n}$ порождает $\Lambda_{0}$-модуль $\Lambda_{1} \otimes_{\Lambda_{0}} \Lambda_{1}$. Действительно, для $u, v \in \Lambda_{1}$ имеет место

$$
\begin{aligned}
& u \otimes v=\left(\left(x_{1} y_{1}+\cdots+x_{n} y_{n}\right) u \otimes v\right)=x_{1} y_{1} u \otimes v+\cdots+x_{n} y_{n} u \otimes v \\
& =x_{0} \otimes y_{1} u v+\cdots+x_{n} \otimes y_{n} u v=u v\left(x_{1} \otimes y_{1}+\cdots+x_{n} \otimes y_{n}\right) .
\end{aligned}
$$

Тогда отображение $\sum_{i} u_{i} \otimes v_{i} \mapsto \sum_{i} u_{i} v_{i}$ является изоморфизмом $\Lambda_{0}$-модулей $\Lambda_{1} \otimes_{\Lambda_{0}} \Lambda_{1}$ и $\Lambda_{0}$. Поэтому $\Lambda_{1}-$ проективный $\Lambda_{0}$-модуль ранга 1.

Лемма доказана. 
Обобщенная скобка Лейбница $\{$,$\} , заданная на супералгебре \Gamma=\Gamma_{0}+\Gamma_{1}$, называется обобщенной скобкой Пуассона (см. [29]), если для $a, b, c \in \Gamma_{0} \cup \Gamma_{1}$ выполняется равенство

$$
\{a,\{b, c\}\}+(-1)^{|a|(|b|+|c|)}\{b,\{c, a\}\}+(-1)^{|c|(|a|+|b|)}\{c,\{a, b\}\}=0,
$$

т. е. $(\Gamma,\{\}$,$) является супералгеброй Ли. Если при этом D=0$, то $\{$,$\} называ-$ ется скобкой Пуассона.

Супералгебра $J=J_{0}+J_{1}$ называется йордановой супералгеброй, когда ее грассманова оболочка $G(J)$ - йорданова алгебра, т. е. в $G(J)$ выполняются тождества

$$
x y=y x, \quad\left(x^{2} y\right) x=x^{2}(y x) .
$$

Если дубль Кантора $J(\Gamma,\{\}$,$) , построенный по скобке \{$,$\} , является йор-$ дановой супералгеброй, то скобка $\{$,$\} называется йордановой скобкой.$

Скобка $\{$,$\} , заданная на унитальной супералгебре \Gamma=\Gamma_{0}+\Gamma_{1}$, является йордановой (см. $[1,2])$ тогда и только тогда, когда выполнены следующие соотношения:

$$
\begin{gathered}
\{a, b c\}=\{a, b\} c+(-1)^{|a||b|} b\{a, c\}-\{a, 1\} b c, \\
\{a, b\}\{c, 1\}+(-1)^{|a||b|+|a||c|}\{b, c\}\{a, 1\}+(-1)^{|a||c|+|b||c|}\{c, a\}\{b, 1\} \\
=\{a,\{b, c\}\}+(-1)^{|a||b|+|a||c|}\{b,\{c, a\}\}+(-1)^{|a||c|+|b||c|}\{c,\{a, b\}\}, \\
\{\{d, d\}, d\}=-\{d, d\}\{d, 1\},
\end{gathered}
$$

где $a, b, c \in \Gamma_{0} \cup \Gamma_{1}, d \in \Gamma_{1}$.

Таким образом, скобка йорданова, если $J(a, b, c)=S(a, b, c)$ для $a, b, c \in$ $\Gamma_{0} \cup \Gamma_{1}$.

В силу (6) отображение $D: a \mapsto\{a, 1\}$ - дифференцирование алгебры $Г$. Тогда (6) эквивалентно

$$
\{a, b c\}=\{a, b\} c+(-1)^{|a||b|} b\{a, c\}-D(a) b c,
$$

т. е. йорданова скобка $\{$,$\} - обобщенная скобка Лейбница.$

Если $\{$,$\} - йорданова скобка, то J(1, a, b)=S(1, a, b)=0$ для любых $a, b \in$ $\Gamma$. Поэтому $D$ - дифференцирование супералгебры $(\Gamma,\{\}$,$) .$

Йорданова скобка называется скобкой векторного типа, если

$$
\{a, b\}=D(a) b-a D(b)
$$

для любых $a, b \in \Gamma$. Йорданову супералгебру скобки векторного типа обозначим через $J(\Gamma, D)$.

Йорданова скобка называется скобкой пуассонова типа, если $D(a)=0$ для любого $a \in \Gamma$.

Пусть $J=A+M-$ йорданова супералгебра с четной частью $A$ и нечетной частью $M$. Пусть $B=B_{0}+B_{1}$ - ассоциативная супералгебра с операцией умножения $*$. Определив на пространстве $B$ суперсимметрическое произведение

$$
a \circ_{s} b=\frac{1}{2}\left(a * b+(-1)^{|a||b|} b * a\right), \quad a, b \in B_{0} \cup B_{1},
$$

получим йорданову супералгебру $B^{+}$. Йорданова супералгебра $J$ называется специальной, если она вкладывается (как $Z_{2}$-градуированная алгебра) в супералгебру $B^{+}$для подходящей ассоциативной супералгебры $B$. Супералгебра $J$ называется исключителъной, если $J$ не является специальной. 


\section{$\S 2$. Обобщенные скобки Лейбница, определенные на координатной алгебре $n$-мерной сферы}

Пусть $F\left[x_{0}, \ldots, x_{n}\right]$ - алгебра полиномов от переменных $x_{0}, x_{1}, \ldots, x_{n}$. Рассмотрим многочлен $S^{n}\left(x_{0}, \ldots, x_{n}\right)=x_{0}^{2}+\cdots+x_{n}^{2}-1$. Пусть

$$
\Lambda(n)=F\left[x_{0}, \ldots, x_{n}\right] /\left(S^{n}\left(x_{0}, \ldots, x_{n}\right)\right)
$$

- фактор-алгебра алгебры $F\left[x_{0}, \ldots, x_{n}\right]$ по идеалу $\left(S^{n}\left(x_{0}, \ldots, x_{n}\right)\right)$, порожденному многочленом $S^{n}\left(x_{0}, \ldots, x_{n}\right)$. Отождествим образы переменных $x_{0}, x_{1}, \ldots$, $x_{n}$ при каноническом гомоморфизме $F\left[x_{0}, \ldots, x_{n}\right] \mapsto \Lambda(n)$ соответственно с $x_{0}, x_{1}, \ldots, x_{n}$. Пусть $\Lambda(n)_{0}$ - подалгебра $\Lambda(n)$, порожденная мономами четной степени, а $\Lambda(n)_{1}=\Lambda(n)_{0} x_{0}+\cdots+\Lambda(n)_{0} x_{n}$. Тогда $\Lambda(n)=\Lambda(n)_{0}+\Lambda(n)_{1}$ является $Z_{2}$-градуированной алгеброй. Произведение элементов $a, b$ в алгебре $\Lambda(n)$ будем обозначать через $a b$. Алгебра $\Lambda(n)$ не имеет делителей нуля (см., например, [30]). По лемме $5 \Lambda(n)_{1}$ - проективный $\Lambda(n)_{0}$-модуль ранга 1.

Лемма 6. Пусть $\{$,$\} - обобщенная скобка Лейбница, заданная на \Lambda(1)$. Тогда $\{$,$\} - йорданова скобка векторного типа относительно дифференци-$ рования $D: a \mapsto\{a, 1\}$. Если характеристика поля $F$ равна нулю, то для $D=x_{1} \frac{\partial}{\partial x_{0}}-x_{0} \frac{\partial}{\partial x_{1}}$ йорданова супералгебра $J\left(\Lambda(1)_{0}, \Lambda(1)_{1},\{\},\right)$ простая. Если уравнение $t^{2}+1=0$ неразрешимо в $F$, то $\Lambda(1)_{0}$-модуль $\Lambda(1)_{1}$ не порождается одним элементом, т. е. не изоморфен $\Lambda(n)_{0}$.

ДокАЗАтеЛьство. Пусть $\{$,$\} - обобщенная скобка Лейбница, заданная$ на алгебре $\Lambda(1)$ и $x=x_{0}, y=x_{1}$. Тогда для любого $a \in \Lambda(1)$ получаем

$$
D(a)=\{a, 1\}=\left\{a, x^{2}+y^{2}\right\}=2\{a, x\} x+2\{a, y\} y-D(a) .
$$

Следовательно,

$$
D(a)=\{a, x\} x+\{a, y\} y .
$$

Поэтому

$$
\left\{\begin{array}{l}
D(x)=\{x, y\} y \\
D(y)=\{y, x\} x
\end{array}\right.
$$

Отсюда

$$
\{x, y\}=\{x, y\} x^{2}+\{x, y\} y^{2}=D(x) y-x D(y) .
$$

Тогда в силу (1) $\{$,$\} является скобкой векторного типа.$

Пусть $D=y \frac{\partial}{\partial x}-x \frac{\partial}{\partial y}$. Тогда $D-$ четное дифференцирование $Z_{2}$-градуированной алгебры $\Lambda(1)$. Поэтому скобка

$$
\{a, b\}=D(a) b-a D(b)
$$

согласована с $Z_{2}$-градуировкой алгебры $\Lambda(1)=\Lambda(1)_{0}+\Lambda(1)_{1}$. Положим $D_{11}=$ $x^{2} D, D_{22}=y^{2} D, D_{12}=x y D$. Выполняются равенства

$$
\begin{gathered}
\{a x, b x\}=D_{11}(a) b-a D_{11}(b), \quad\{a y, b y\}=D_{22}(a) b-a D_{22}(b), \\
\{a x, b y\}=a b+D_{12}(a) b-a D_{12}(b),
\end{gathered}
$$

где $a, b \in \Lambda(1)_{0}$. Если характеристика поля $F$ равна нулю, то, как показано в $[19,20]$, супералгебра $J\left(\Lambda(1)_{0}, \Lambda(1)_{1},\{\},\right)$ простая. Там же показано, что $\Lambda(1)_{0}$-модуль $\Lambda(1)_{1}$ не порождается одним элементом, если уравнение $t^{2}+1=0$ неразрешимо в $F$. 
Лемма 7. Любая йорданова скобка, заданная на $\Lambda(2)$, является обобщенной скобкой Пуассона. Пусть $\{$,$\} - обобщенная скобка Лейбница, заданная на$ $\Lambda(2)$. Тогда следующие условия эквивалентны:

(i) $\{$,$\} - йорданова скобка,$

(ii) $D: a \mapsto\{a, 1\}$ - дифференцирование алгебры $(\Lambda(2),\{\}$,$) ,$

(iii) $J\left(1, x_{i}, x_{j}\right)=0$ для некоторых $i \neq j$.

ДоказАтЕЛьство. Пусть $\{$,$\} - обобщенная скобка Лейбница, заданная$ на $\Lambda(2)$, и $x=x_{0}, y=x_{1}, z=x_{2}$. В силу леммы 1

$$
\begin{aligned}
S(1, x, y)=S\left(x^{2}+y^{2}\right. & \left.+z^{2}, x, y\right) \\
& =2 S(x, x, y) x+2 S(y, x, y) y+2 S(z, x, y) z=2 S(x, y, z) x .
\end{aligned}
$$

Поскольку $S(1, x, y)=0$, то $S(x, y, z) x=0$. Аналогично $S(x, y, z) y=S(x, y, z) z$ $=0$. Поэтому

$$
S(x, y, z)=S(x, y, z) x^{2}+S(x, y, z) y^{2}+S(x, y, z) z^{2}=0 .
$$

Следовательно, по лемме $1 S(a, b, c)=0$ для любых $a, b, c \in \Lambda(2)$.

Отсюда получаем, что любая йорданова скобка, заданная на $\Lambda(2)$, является обобщенной скобкой Пуассона, т. е. $J(a, b, c)=0$ для любых $a, b, c \in \Lambda(2)$.

Пусть имеет место (i). Тогда $J(1, a, b)=S(1, a, b)$ для любых $a, b \in \Lambda(2)$. Так как $S(1, a, b)=0$, то $J(1, a, b)=0$. Поэтому выполняется (ii).

Ясно, что (ii) влечет (iii).

Покажем, что

$$
J(x, y, z) x=J(1, y, z)=-D(\{y, z\})+\{y, D(z)\}+\{D(y), z\} .
$$

В силу леммы 1 получаем

$$
J(1, y, z)=J\left(x^{2}+y^{2}+z^{2}, y, z\right)=2 x J(x, y, z)-J(1, y, z)\left(x^{2}+y^{2}+z^{2}\right) .
$$

Поэтому $J(1, y, z)=x J(x, y, z)$. Аналогично $J(1, x, y)=z J(x, y, z), J(1, x, z)=$ $-y J(x, y, z)$.

Пусть имеет место (iii). Рассмотрим случай $J(1, y, z)=0$. Тогда $J(x, y, z) x$ $=0$. Поскольку алгебра $\Lambda(2)$ не содержит делителей нуля, то $J(x, y, z)=0$. Поэтому в силу леммы $1 J(a, b, c)=0$ для любых $a, b, c \in \Lambda(2)$. Таким образом, $\{$,$\} - йорданова скобка на \Lambda(2)$, т. е. выполняется (i).

Следствие 1. Любое решение $u_{1}, u_{2}, u_{3}$ системы уравнений

$$
\left\{\begin{array}{l}
u_{1} y+u_{2} z=0 \\
u_{1} x-u_{3} z=0 \\
u_{2} x+u_{3} y=0
\end{array}\right.
$$

в алгебре $\Lambda(2)$ определяет на $\Lambda(2)$ йорданову скобку пуассонова типа. $B$ частности, любая скобка Лейбница, заданная на $\Lambda(2)$, является йордановой скобкой пуассонова типа. Любая йорданова скобка на $\Lambda(2)$ является суммой йордановых скобок векторного типа и пуассонова типа.

ДокАзАтЕЛьство. Пусть $\{$,$\} - йорданова скобка на \Lambda(2)$. Тогда справедливо (6) и $D: a \mapsto\{a, 1\}$ - дифференцирование алгебры $\Lambda(2)$. Определим на $\Lambda(2)$ новую скобку, полагая

$$
\langle a, b\rangle=\{a, b\}-D(a) b+a D(b) .
$$

Тогда $\langle$,$\rangle - скобка Лейбница и в силу леммы 7\langle$,$\rangle - йорданова скобка пуас-$ сонова типа. Отсюда получаем, что йорданова скобка $\{$,$\} является суммой$ скобки векторного типа и йордановой скобки пуассонова типа. 
Теорема 2. Для любого $n \geq 1$ на алгебре $\Lambda(n)$ можно задать йорданову скобку $\{,\}_{n}$, согласованную с $Z_{2}$-градуировкой алгебры $\Lambda(n)=\Lambda(n)_{0}+\Lambda(n)_{1}$, для которой справедливы следующие утверждения.

1. Если $n=1$, то $\{a, b\}_{1}=D(a) b-a D(b)$ для $a, b \in \Lambda(1)$, где $D=x_{1} \frac{\partial}{\partial x_{0}}-$ $x_{0} \frac{\partial}{\partial x_{1}}$.

2. Пусть $k$ - четное число, $k \leq n$ и $z(k)=\sum_{i=0}^{k}(-1)^{i} x_{i}$. Тогда $\left\{z(k), x_{i}\right\}_{n}=0$ для $i \leq k$.

3. Элемент $z(2)=x_{0}-x_{1}+x_{2}$ принадлежит центру алгебры Ли $\left(\Lambda(2),\{,\}_{2}\right)$.

4. $I=z(2) \Lambda(2)_{1}+z(2) \Lambda(2)_{0} \xi-$ идеал супералгебры $J\left(\Lambda(2)_{0}, \Lambda(2)_{1},\{,\}_{2}\right)$.

ДокАЗАТЕЛЬСТвО. По определению йордановой скобки положим $\{a, b\}_{n}=$ $-\{b, a\}_{n}$ для любых $a, b \in \Lambda(n)$. На порождающих $1, x_{0}, \ldots, x_{n}$ скобку $\{,\}_{n}$ и дифференцирование $D$ зададим следующим образом:

$$
\begin{gathered}
\left\{x_{i}, x_{j}\right\}_{n}=1, \quad 0 \leq i<j \leq n, \\
D\left(x_{i}\right)_{n}=\left\{x_{i}, 1\right\}_{n}=-\sum_{k=0}^{i-1} x_{k}+\sum_{k=i+1}^{n} x_{k}, \quad i=0, \ldots, n .
\end{gathered}
$$

Используя (1), скобку $\{,\}_{n}$ можно продолжить на всю алгебру $\Lambda(n)$. Скобка $\{,\}_{n}$ задана корректно.

Покажем, что выполняется (7). В силу леммы 1 равенство (7) достаточно проверить для порождающих алгебры $\Lambda(n)$. Пусть $0 \leq i<j<k \leq n$. Тогда

$$
J\left(x_{i}, x_{j}, x_{k}\right)=\left\{x_{i}, 1\right\}_{n}-\left\{x_{j}, 1\right\}_{n}+\left\{x_{k}, 1\right\}_{n}=D\left(x_{i}\right)-D\left(x_{j}\right)+D\left(x_{k}\right) .
$$

С другой стороны,

$$
S\left(x_{i}, x_{j}, x_{k}\right)=D\left(x_{k}\right)+D\left(x_{i}\right)-D\left(x_{j}\right) .
$$

Следовательно, $J\left(x_{i}, x_{j}, x_{k}\right)=S\left(x_{i}, x_{j}, x_{k}\right)$. Если $i=j$, то

$$
J\left(x_{i}, x_{i}, x_{k}\right)=S\left(x_{i}, x_{i}, x_{k}\right)=0 .
$$

В силу леммы 1

$$
J\left(1, x_{j}, x_{k}\right)=J\left(\sum_{i=0}^{n} x_{i}^{2}, x_{j}, x_{k}\right)=\sum_{i=0}^{n} 2 x_{i} J\left(x_{i}, x_{j}, x_{k}\right)-J\left(1, x_{j}, x_{k}\right) .
$$

Поэтому $J\left(1, x_{j}, x_{k}\right)=\sum_{i=0}^{n} x_{i} J\left(x_{i}, x_{j}, x_{k}\right)$. По доказанному

$$
J\left(x_{i}, x_{j}, x_{k}\right)=S\left(x_{i}, x_{j}, x_{k}\right) .
$$

Следовательно, в силу леммы 1

$$
J\left(1, x_{j}, x_{k}\right)=\sum_{i=0}^{n} x_{i} J\left(x_{i}, x_{j}, x_{k}\right)=\sum_{i=0}^{n} x_{i} S\left(x_{i}, x_{j}, x_{k}\right)=\frac{1}{2} S\left(1, x_{j}, x_{k}\right) .
$$

Поскольку $S\left(1, x_{j}, x_{k}\right)=0$, то $J\left(1, x_{j}, x_{k}\right)=0$.

Таким образом, имеет место (7), т. е. $\{,\}_{n}$ - йорданова скобка. Ясно, что скобка $\{,\}_{n}$ согласована с $Z_{2}$-градуировкой алгебры $\Lambda(n)$.

В силу леммы 6 имеет место п. 1. 
Докажем п. 2. Пусть $i \leq k$. Тогда

$$
\left\{z(k), x_{i}\right\}_{n}=\sum_{j=0}^{i-1}(-1)^{j}+\sum_{j=i+1}^{k}-(-1)^{j} .
$$

Если $i$ - четное число, то $\sum_{j=0}^{i-1}(-1)^{j}=0$ и $\sum_{j=i+1}^{k}-(-1)^{j}=0$. Тогда $\left\{z(k), x_{i}\right\}_{n}=$ 0. Если $i-$ нечетное число, то $\sum_{j=0}^{i-1}(-1)^{j}=1$ и $\sum_{j=i+1}^{k}-(-1)^{j}=-1$. Тогда $\left\{z(k), x_{i}\right\}_{n}=0$.

Докажем п. 3. В силу леммы $7\left(\Lambda(2),\{,\}_{2}\right)$ - алгебра Ли. В силу п. 2 $D(z(2))=\{z(2), 1\}_{2}=0$ и $\left\{z(2), x_{i}\right\}_{2}=0$, где $i=0,1,2$. Поэтому в силу (1) отображение

$$
\varphi: a \in \Lambda(2) \mapsto\{z(2), a\}_{2}
$$

является дифференцированием алгебры $\Lambda(2)$. Так как $\varphi(1)=\varphi\left(x_{i}\right)=0$ для $i=0,1,2$, то $\varphi=0$. Следовательно, $\{z(2), \Lambda(2)\}_{2}=0$, т. е. $z(2)$ принадлежит центру алгебры Ли $\left(\Lambda(2),\{,\}_{2}\right)$.

Докажем п. 4. Пусть

$$
I=z(2) \Lambda(2)_{1}+z(2) \Lambda(2)_{0} \xi
$$

В силу определения операции умножения в супералгебре $J\left(\Lambda(2)_{0}, \Lambda(2)_{1},\{,\}_{2}\right)$ получаем

$$
I \cdot \Lambda(2)_{0} \subseteq z(2) \Lambda(2)_{1} \Lambda(2)_{0}+\left(z(2) \Lambda(2)_{0} \Lambda(2)_{0}\right) \xi \subseteq I .
$$

Аналогично

$$
z(2) \Lambda(2)_{1} \cdot \Lambda(2)_{1} \xi \subseteq\left(z(2) \Lambda(2)_{1} \Lambda(2)_{1}\right) \xi \subseteq z(2) \Lambda(2)_{0} \xi \subseteq I .
$$

В силу (1) и п. 3

$$
\begin{aligned}
\Lambda(2)_{1} \xi \cdot z(2) \Lambda(2)_{0} \xi \subseteq\left\{\Lambda(2)_{1}, z(2) \Lambda(2)_{0}\right\}_{2} \\
\\
\left.\subseteq z(2)\left\{\Lambda(2)_{1}, \Lambda(2)_{0}\right\}_{2}+D\left(\Lambda(2)_{1}\right) z(2) \Lambda(2)_{0} \subseteq z(2)\right) \Lambda(2)_{1} \subseteq I .
\end{aligned}
$$

Следовательно, $I$ - идеал супералгебры $J\left(\Lambda(2)_{0}, \Lambda(2)_{1},\{,\}_{2}\right)$.

Предположим, что $I=J\left(\Lambda(2)_{0}, \Lambda(2)_{1},\{,\}_{2}\right)$. Тогда

$$
z(2) t=\left(x_{0}-x_{1}+x_{2}\right) t=1
$$

для некоторого $t \in \Lambda(2)_{1}$. Поскольку $\Lambda(2)=F\left[x_{0}, x_{1}\right] \oplus x_{2} F\left[x_{0}, x_{1}\right]$, то $t=f+x_{2} g$, где $f, g \in F\left[x_{0}, x_{1}\right]$, откуда

$$
\left(x_{0}-x_{1}\right) f+\left(1-x_{0}^{2}-x_{1}^{2}\right) g=1, f+\left(x_{0}-x_{1}\right) g=0 .
$$

Следовательно,

$$
\left(\left(1-x_{0}^{2}-x_{1}^{2}\right)-\left(x_{0}-x_{1}\right)^{2}\right) g=1 .
$$

Отсюда получаем, что $1-2 x_{0}^{2}-2 x_{1}^{2}+2 x_{0} x_{1}$ обратим в $F\left[x_{0}, x_{1}\right]$. Поэтому $I-$ собственный идеал в $J\left(\Lambda(2)_{0}, \Lambda(2)_{1},\{,\}_{2}\right)$. 
Лемма 8. Йорданова супералгебра $J\left(\Lambda(2)_{0}, \Lambda(2)_{1},\{,\}_{2}\right)$ исключительна. $B$ частности, $J\left(\Lambda(2),\{,\}_{2}\right)$ - исключительная йорданова супералгебра.

ДокАЗАТЕЛЬСтво. Положим $x=x_{0}, y=x_{1}, z=x_{2}, u=x-y+z$. Определим на $\Lambda(2)$ скобку $\langle$,$\rangle , полагая \langle a, b\rangle=\{a, b\}_{2}-D(a) b+a D(b)$, где $D$ - дифференцирование скобки $\{,\}_{2}$. Тогда $\left\langle\Lambda(2)_{0}, \Lambda(2)_{0}\right\rangle \subseteq \Lambda(2)_{0}$ и для $a, b, c \in \Lambda(2)$ имеет место $\langle a, b c\rangle=b\langle a, c\rangle+\langle a, b\rangle$.

Пусть $J\left(\Lambda(2)_{0}, \Lambda(2)_{1},\{,\}_{2}\right)$ - специальная супералгебра. Тогда подсупералгебра $J=\Lambda(2)_{0}+\left(\Lambda(2)_{0} u\right) \xi$ специальна и является супералгеброй йордановой скобки $\{a, b\}=\{a u, b u\}_{2}$ на алгебре $\Lambda(2)_{0}$. В силу теоремы $2\{\Lambda(2), u\}_{2}=0$. Поэтому $\{\}-$, скобка пуассонова типа. В силу работы [7] для $a, b, c \in \Lambda(2)_{0}$ выполняется тождество $\{\{a, b\}, c\}=0$. Так как $\{\Lambda(2), u\}_{2}=0$, в силу (1) для $a, b \in \Lambda(2)_{0}$

$$
\{a, b\}=\{a u, b u\}_{2}=\{a, b\}_{2} u^{2}-D(a) b u^{2}+a D(b) u^{2}=\langle a, b\rangle u^{2} .
$$

Следовательно, для $a, b, c \in \Lambda(2)_{0}$

$$
\begin{aligned}
0=\{\{a, b\}, c\}= & \left\langle\langle a, b\rangle u^{2}, c\right\rangle u^{2} \\
& =\langle\langle a, b\rangle, c\rangle u^{4}+\langle a, b\rangle\left\langle u^{2}, c\right\rangle u^{2}=\langle\langle a, b\rangle, c\rangle u^{4}+2\langle a, b\rangle D(c) u^{4} .
\end{aligned}
$$

Поэтому $\langle\langle a, b\rangle, c\rangle=-2\langle a, b\rangle D(c)$ для $a, b, c \in \Lambda(2)_{0}$.

Пусть $a=x^{2}, b=y^{2}, c=z^{2}$. Тогда

$$
\langle a, b\rangle=4 x y\langle x, y\rangle=4 x y(1-(y+z) y+x(-x+z))=4 x y z u .
$$

Поэтому

$$
\begin{gathered}
\langle\langle a, b\rangle, c\rangle=\left\langle 4 x y z u, z^{2}\right\rangle=8 z^{2}\langle x y u, z\rangle=8 z^{2} u(x\langle y, z\rangle+\langle x, z\rangle y)+8 x y z^{2}\langle u, z\rangle \\
=8 z^{2} u(x(1-(-x+z) z+y(-x-y)))+y(1-(y+z) z+x(-x-y))+8 x y z^{2} u D(z) \\
=8 z^{2} u^{2}\left(x^{2}-y^{2}\right)-8 x y z^{2} u(x+y) .
\end{gathered}
$$

$\mathrm{C}$ другой стороны, $2\langle a, b\rangle D(c)=-16 x y z^{2} u(x+y)$. Следовательно,

$$
8 z^{2} u^{2}\left(x^{2}-y^{2}\right)-8 x y z^{2} u(x+y)=16 x y z^{2} u(x+y) .
$$

Поскольку алгебра $\Lambda(2)$ не имеет делителей нуля, то $u(x-y)=3 x y$; противоречие. Стало быть, $J=\Lambda(2)_{0}+\left(\Lambda(2)_{0} u\right) \xi-$ исключительная супералгебра.

Таким образом, $J\left(\Lambda(2)_{0}, \Lambda(2)_{1},\{,\}_{2}\right)$ - исключительная супералгебра. Так как $J\left(\Lambda(2)_{0}, \Lambda(2)_{1},\{,\}_{2}\right)$ - подсупералгебра супералгебры $J\left(\Lambda(2),\{,\}_{2}\right)$, то $J\left(\Lambda(2),\{,\}_{2}\right)$ - исключительная супералгебра.

Лемма 9. При $n \geq 3$ на алгебре $\Lambda(n)$ можно задать скобку Лейбница, которая не является йордановой.

ДокАЗАТЕЛЬСТво. Рассмотрим на $\Lambda(n)$ йорданову скобку, определенную в теореме 2. А именно,

$$
\begin{gathered}
\left\{x_{i}, x_{i}\right\}_{n}=0, \quad\left\{x_{i}, x_{j}\right\}_{n}=1, \quad 0 \leq i<j \leq n, \\
D\left(x_{i}\right)_{n}=\left\{x_{i}, 1\right\}_{n}=-\sum_{k=0}^{i-1} x_{k}+\sum_{k=i+1}^{n} x_{k}, \quad i=0, \ldots, n .
\end{gathered}
$$

Пусть $J(a, b, c)_{n}$ - якобиан скобки $\{,\}_{n}$ и $S(a, b, c)_{n}-$ функция $S$ скобки $\{,\}_{n}$, определенная равенством (2). 
Определим на $\Lambda(n)$ скобку Лейбница

$$
\langle a, b\rangle=\{a, b\}-D(a) b+D(b) a .
$$

Через $J(a, b, c)_{\langle,\rangle}$и $S(a, b, c)_{\langle,\rangle}$обозначим якобиан и функцию $S$ скобки $\langle$,$\rangle . Так$ как $\{,\}_{n}$ - йорданова скобка, то $J(1, a, b)_{n}=0$ для любых $a, b \in \Lambda(n)$. По лемме 2

$$
J(a, b, c)_{\langle,\rangle}=J(a, b, c)_{n}+S(a, b, c)_{n} .
$$

Следовательно, при $0 \leq i<j<k \leq n$

$$
J\left(x_{i}, x_{j}, x_{k}\right)_{\langle,\rangle}=2\left(D\left(x_{i}\right)-D\left(x_{j}\right)+D\left(x_{k}\right)\right) .
$$

Подставив значения $D\left(x_{i}\right), D\left(x_{j}\right), D\left(x_{k}\right)$, получим

$$
\begin{aligned}
D\left(x_{i}\right)-D\left(x_{j}\right)+D\left(x_{k}\right)=-\sum_{l=0}^{i-1} x_{l}+ & \sum_{l=i+1}^{n} x_{l}+\sum_{l=0}^{j-1} x_{l}-\sum_{l=j+1}^{n} x_{l}-\sum_{l=0}^{k-1} x_{l}+\sum_{l=k+1}^{n} x_{l} \\
& =-\sum_{l=0}^{i-1} x_{l}+\sum_{l=i+1}^{j-1} x_{l}-\sum_{l=j+1}^{k-1} x_{l}+\sum_{l=k+1}^{n} x_{l} .
\end{aligned}
$$

Следовательно, $J\left(x_{i}, x_{j}, x_{k}\right)_{\langle,\rangle} \neq 0$.

С другой стороны, $\langle a, 1\rangle=0$ для $a \in \Lambda(n)$, т. е. $S\left(x_{i}, x_{j}, x_{k}\right)_{\langle,\rangle}=0$. Поэтому $J\left(x_{i}, x_{j}, x_{k}\right)_{\langle,\rangle} \neq S\left(x_{i}, x_{j}, x_{k}\right)_{\langle,\rangle}$и $\langle$,$\rangle не является йордановой скобкой.$

\section{$\S$ 3. Простые абелевы йордановы супералгебры, определенные $\boldsymbol{n}$-сферой}

Супералгебра $J=A+M$ называется абелевой, если $A-$ ассоциативная алгебра, $M$ - ассоциативный $A$-модуль. В [22] доказано следующее утверждение.

Пусть $J=A+M$ - простая абелева йорданова супералгебра, не изоморфная супералгебре билинейной формы. Положим $D_{x, y}(a)=(a x) y-a(x y)$, где $a \in A, x, y \in M$. Тогда алгебра $A$ дифференциально проста относительно множества дифференцирований $\Delta=\left\{D_{x, y} \mid x, y \in M\right\}$ алгебры $A$. Более того, $J-$ унитальная супералгебра, а $M$ - конечнопорожденный проективный $A$-модуль ранга 1.

В [18] доказана

Теорема 3. Пусть $J=A+M$ - простая унитальная специальная йорданова абелева супералгебра, не изоморфная супералгебре билинейной формы. Тогда существуют такие элементы $x_{1}, \ldots, x_{n} \in M$, что

$$
M=A x_{1}+\cdots+A x_{n}
$$

и произведение в $M$ задается равенством

$$
a x_{i} \cdot b x_{j}=\gamma_{i j} a b+D_{i j}(a) b-a D_{j i}(b), \quad i, j=1, \ldots, n,
$$

где $\gamma_{i j} \in A$, а $D_{i j}$ - дифференцирование алгебры $A$. Алгебра $A$ дифференциально простая относительно множества дифференцирований $\Delta=\left\{D_{i j} \mid i, j=\right.$ $1, \ldots, n\}$. Кроме того, супералгебра $J$ является подалгеброй йордановой супералгебры векторного типа $J(\Gamma, D)$.

В [18-21] построены примеры простых унитальных специальных йордановых абелевых супералгебр, нечетная часть которых порождается как модуль двумя элементами и не порождается одним элементом. Поэтому построенные примеры супералгебр не изоморфны супералгебре векторного типа $J(\Gamma, D)$.

В этом параграфе будут построены примеры простых унитальных исключительных йордановых абелевых супералгебр $J=A+M$, четная часть которых как $A$-модуль имеет произвольное число порождающих. 
Теорема 4. Пусть $F-$ поле характеристики 0 и $n-$ нечетное число. Тогда йорданова супералгебра $J\left(\Lambda(n),\{,\}_{n}\right)$ простая. $B$ частности, $J\left(\Lambda(n)_{0}, \Lambda(n)_{1}\right.$, $\left.\{,\}_{n}\right)$ является простой супералгеброй.

Доказательство. Обозначим скобку $\{,\}_{n}$ через $\{$,$\} . По теореме 2$

$$
\begin{gathered}
\left\{x_{i}, x_{i}\right\}=0, \quad\left\{x_{i}, x_{j}\right\}=1, \quad 0 \leq i<j \leq n, \\
D\left(x_{i}\right)=-\sum_{j=0}^{i-1} x_{j}+\sum_{j+1}^{n} x_{j}, \quad i=0, \ldots, n .
\end{gathered}
$$

Пусть $D_{u, v}(a)=\{a u, v\}-a\{u, v\}$ и $\mathscr{D}=\left\{D_{u, v} \mid u, v \in \Lambda(n)\right\}$. По лемме 3 достаточно доказать, что $\Lambda(n)$ дифференциально проста относительно дифференцирований из $\mathscr{D}$.

Предположим, что алгебра $\Lambda(n)$ не является дифференциально простой. Тогда множество идеалов алгебры $\Lambda(n)$, инвариантных относительно дифференцирований из $\mathscr{D}$, содержит максимальный идеал $I$. В силу [31] $I$ - простой идеал. Так как $D_{u, v}(I) \in I$ для любых $u, v \in \Lambda(n)$, имеем

$$
\{I, u\} \subseteq D_{1, u}(I)+I\{1, u\} \subseteq I .
$$

Поэтому $\{I, \Lambda(n)\} \subseteq I$. Заметим, что $x_{i} \notin I$, иначе $\left\{x_{i}, x_{j}\right\}= \pm 1 \in I$ и $I=\Lambda(n)$.

Поскольку $\Lambda(n)=F\left[x_{0}, \ldots, x_{n-1}\right]+x_{n} F\left[x_{0}, \ldots, x_{n-1}\right]$ и алгебра $\Lambda(n)$ не имеет делителей нуля, то $F\left[x_{0}, \ldots, x_{n-1}\right] \cap I \neq 0$.

Пусть $u \in \Lambda(n)$. В силу (1) отображение $d: a \mapsto\{u, a\}-D(u) a$ является дифференцированием алгебры $\Lambda(n)$. Тогда

$$
\begin{aligned}
d\left(x_{0}^{i_{0}} \ldots x_{n-1}^{i_{n-1}}\right)=\sum_{j=0}^{n-1} d\left(x_{j}^{i_{j}}\right) x_{0}^{i_{0}} \ldots & x_{j-1}^{i_{j-1}} x_{j+1}^{i_{j+1}} \ldots x_{n-1}^{i_{n-1}} \\
& =\sum_{j=0}^{n-1} i_{j} d\left(x_{j}\right) x_{0}^{i_{0}} \ldots x_{j-1}^{i_{j-1}} x_{j}^{i_{j}-1} x_{j+1}^{i_{j+1}} \ldots x_{n-1}^{i_{n-1}} .
\end{aligned}
$$

Поэтому

$$
\begin{aligned}
\left\{u, x_{0}^{i_{0}} \ldots x_{n-1}^{i_{n-1}}\right\}=\sum_{j=0}^{n-1} i_{j}\left\{u, x_{j}\right\} x_{0}^{i_{0}} \ldots x_{j-1}^{i_{j-1}} x_{j}^{i_{j}-1} x_{j+1}^{i_{j+1}} \ldots x_{n-1}^{i_{n-1}} & \\
& -D(u)\left(\sum_{j=0}^{n-1} i_{j}-1\right) x_{0}^{i_{0}} \ldots x_{n-1}^{i_{n-1}} .
\end{aligned}
$$

Пусть $f \in F\left[x_{0}, x_{1}, \ldots, x_{n-1}\right] \cap I, f \neq 0$. Тогда $f=f_{k}+f_{k-1}+\cdots+f_{0}$, где каждый ненулевой $f_{i}$ - однородный многочлен из $F\left[x_{0}, x_{1}, \ldots, x_{n-1}\right]$ степени $i$, $i=0, \ldots, k$. Пусть многочлен $f$ имеет наименьшую степень $k$.

Предположим, что $k>0$. Пусть $z(n-1)=\sum_{i=0}^{n-1}(-1)^{i} x_{i}$. По теореме 2, п. 2 , имеем $\left\{z(n-1), x_{i}\right\}=\left\{z(n-1), x_{i}\right\}_{n}=0, i=0, \ldots, n-1$. Тогда

$$
D(z(n-1))=\{z(n-1), 1\}=\sum_{i=0}^{n}\left\{z(n-1), x_{i}\right\} x_{i}=\left\{z(n-1), x_{n}\right\} x_{n}=x_{n} .
$$

Следовательно,

$$
\left\{z(n-1), x_{0}^{i_{0}} \ldots x_{n-1}^{i_{n-1}}\right\}=-x_{n}\left(\sum_{j=0}^{n-1} i_{j}-1\right) x_{0}^{i_{0}} \ldots x_{n-1}^{i_{n-1}} .
$$


Поэтому $\left\{z(n-1), f_{i}\right\}=-(i-1) x_{n} f_{i}$, где $i=0, \ldots, k$. Отсюда получаем $\{z(n-1), f\}=\left\{z(n-1), f_{k}+f_{k-1}+\cdots+f_{0}\right\}=\left(-(k-1) f_{k}-(k-2) f_{k-1}+\cdots+f_{0}\right) x_{n}$.

Поскольку $\{z(n-1), f\} \in I, I-$ простой идеал и $x_{n} \notin I$, то $g=-(k-1) f_{k}-$ $(k-2) f_{k-1}+\cdots+f_{0} \in I$. Тогда

$$
(k-1) f+g=f_{k-1}+2 f_{k-2}+\cdots+k f_{0} \in F\left[x_{0}, x_{1}, \ldots, x_{n-1}\right] \cap I
$$

и степень многочлена $(k-1) f+g$ меньше, чем $k$. В силу выбора $f$ можно считать, что $f$ - однородный многочлен степени $k$.

Пусть

$$
f=\sum_{i_{0}+\cdots+i_{n-1}=k} \alpha_{i_{0} \ldots i_{n-1}} x_{0}^{i_{0}} \ldots x_{n-1}^{i_{n-1}},
$$

где $\alpha_{i_{0} \ldots i_{n-1}} \in F$. Пусть $x_{j}$ - переменная с наибольшим индексом, входящая в запись многочлена $f$. Не теряя общности, можно считать, что $x_{j}=x_{n-1}$. Положим $u=x_{n-1}-x_{n}$. Тогда $\left\{u, x_{i}\right\}=0, i=0, \ldots, n-2$, и $\left\{u, x_{n-1}\right\}=1$. Так как $f \in I,\{u, f\} \in I$ и

$$
\{u, f\}=\sum_{i_{0}+\cdots+\left(i_{n-1}-1\right)=k-1} i_{n-1} \alpha_{i_{0}, \ldots, i_{n-1}} x_{0}^{i_{0}} \ldots x_{n-2}^{i_{n-2}} x_{n-1}^{i_{n-1}-1}-(k-1) f D(u),
$$

TO

$$
\sum_{i_{0}+\cdots+\left(i_{n-1}-1\right)=k-1} i_{n-1} \alpha_{i_{0}, \ldots, i_{n-1}} x_{0}^{i_{0}} \ldots x_{n-2}^{i_{n-2}} x_{n-1}^{i_{n-1}-1} \in I .
$$

В силу выбора $f$ получаем

$$
\sum_{i_{0}+\cdots+\left(i_{n-1}-1\right)=k-1} i_{n-1} \alpha_{i_{0}, \ldots, i_{n-1}} x_{0}^{i_{0}} \ldots x_{n-2}^{i_{n-2}} x_{n-1}^{i_{n-1}-1}=0 .
$$

Отсюда

$$
\sum_{i_{0}+\cdots+i_{n-2}=k-i_{n-1}} \alpha_{i_{0}, \ldots, i_{n-1}} x_{0}^{i_{0}} \ldots x_{n-2}^{i_{n-2}}=0,
$$

если $i_{n-1} \neq 0$. Поэтому переменная $x_{n-1}$ не входит в запись многочлена $f$; противоречие. Следовательно, $k=0, f \in F$ и $I=\Lambda(n)$.

Таким образом, супералгебра $J\left(\Lambda(n),\{,\}_{n}\right)$ простая. По теореме 1 получаем, что $J\left(\Lambda(n)_{0}, \Lambda(n)_{1},\{,\}_{n}\right)$ - простая супералгебра.

Пусть $X=S^{n} \times \mathbb{A}^{m}$ - декартово произведение $n$-мерной сферы и $m$-мерного аффинного пространства. Тогда $X-$ неприводимое многообразие. Поэтому координатная алгебра $F[X]=\Lambda(n, m)$ многообразия $X$ не имеет делителей нуля. Кроме того,

$$
\Lambda(n, m)=F\left[x_{0}, x_{1}, \ldots, x_{n}, y_{1}, \ldots, y_{m}\right] /\left(S^{n}\left(x_{0}, \ldots, x_{n}\right)\right)
$$

- фактор-алгебра алгебры полиномов $F\left[x_{0}, x_{1}, \ldots, x_{n}, y_{1}, \ldots, y_{m}\right]$ по идеалу $S^{n}\left(x_{0}, \ldots, x_{n}\right) F\left[x_{0}, x_{1}, \ldots, x_{n}, y_{1}, \ldots, y_{m}\right]$. Следовательно, $\Lambda(n, m)=\Lambda(n, m)_{0}+$ $\Lambda(n, m)_{1}-Z_{2}$-градуированная алгебра, где $\Lambda(n, m)_{0}$ - подалгебра в $\Lambda(n, m)$, порожденная мономами четной степени, а $\Lambda(n, m)_{1}=\Lambda(n, m)_{0} x_{0}+\cdots+\Lambda(n, m)_{0} x_{n}$. По лемме $5 \Lambda(n, m)_{1}$ - проективный $\Lambda(n, m)_{0}$-модуль ранга 1 . Заметим, что $\Lambda(n)$ - подалгебра в $\Lambda(n, m)$. 
Лемма 10. Для любого $n \geq 1$ и любого $m$ на алгебре $\Lambda(n, m)$ можно задать йорданову скобку $\{,\}_{n, m}$, согласованную с $Z_{2}$-градуировкой алгебры $\Lambda(n, m)=\Lambda(n, m)_{0}+\Lambda(n, m)_{1}$. Более того, $\left(\Lambda(n),\{,\}_{n}\right)$ является подалгеброй в $\left(\Lambda(n, m),\{,\}_{n, m}\right)$.

ДокАЗАТЕльство. Определим на $\Lambda(n, m)$ скобку $\{,\}_{n, m}$, полагая $\{a, b\}_{n, m}$ $=\{a, b\}_{n}$ на $\Lambda(n)$, а на порождающих $y_{1}, \ldots, y_{m}$

$$
\begin{gathered}
\left\{x_{i}, y_{j}\right\}=1, \quad\left\{y_{j}, y_{j}\right\}=0, \quad\left\{y_{j}, y_{k}\right\}=1, \quad j<k, \\
D\left(y_{j}\right)=\left\{y_{j}, 1\right\}=-\left(x_{0}+\cdots+x_{n}\right), \quad i=0, \ldots, n, j, k=1, \ldots, m .
\end{gathered}
$$

Используя (1), скобку $\{,\}_{n, m}$ можно продолжить на всю алгебру $\Lambda(n, m)$. Скобка $\{,\}_{n, m}$ задана корректно и согласована с $Z_{2}$-градуировкой алгебры $\Lambda(n, m)=\Lambda(n, m)_{0}+\Lambda(n, m)_{1}$. Ясно, что $\left(\Lambda(n),\{,\}_{n}\right)$ является подалгеброй в $\left(\Lambda(n, m),\{,\}_{n, m}\right)$.

Покажем, что выполняется (7). В силу леммы 1 равенство (7) достаточно проверить для порождающих алгебры $\Lambda(n, m)$. По теореме $2 J(a, b, c)=S(a, b, c)$ для $a, b, c \in \Lambda(n)$.

Пусть $0 \leq i<j \leq n$ и $1 \leq k \leq m$. Тогда

$$
J\left(x_{i}, x_{j}, y_{k}\right)=\left\{x_{i}, 1\right\}_{n}-\left\{x_{j}, 1\right\}_{n}+\left\{y_{k}, 1\right\}_{n}=D\left(x_{i}\right)-D\left(x_{j}\right)+D\left(y_{k}\right) .
$$

Но $S\left(x_{i}, x_{j}, y_{k}\right)=D\left(y_{k}\right)+D\left(x_{i}\right)-D\left(x_{j}\right)$. Значит, $J\left(x_{i}, x_{j}, y_{k}\right)=S\left(x_{i}, x_{j}, y_{k}\right)$. Аналогично $J\left(x_{i}, y_{j}, y_{k}\right)=S\left(x_{i}, y_{j}, y_{k}\right), J\left(y_{j}, y_{k}, y_{l}\right)=S\left(y_{j}, y_{k}, y_{l}\right)$ для $0 \leq i \leq n$ и $1 \leq j<k<l \leq m$.

Для $a, b \in \Lambda(n, m)$ в силу леммы 1

$$
J(1, a, b)=J\left(\sum_{i=0}^{n} x_{i}^{2}, a, b\right)=\sum_{i=0}^{n} 2 x_{i} J\left(x_{i}, a, b\right)-J(1, a, b) .
$$

Поэтому $J(1, a, b)=\sum_{i=0}^{n} x_{i} J\left(x_{i}, a, b\right)$. Пусть $a, b \in\left\{x_{0}, \ldots, x_{n}, y_{1}, \ldots, y_{m}\right\}$. Тогда по доказанному $J\left(x_{i}, a, b\right)=S\left(x_{i}, a, b\right), i=0, \ldots, n$. Следовательно, по лемме 1

$$
J(1, a, b)=\sum_{i=0}^{n} x_{i} J\left(x_{i}, a, b\right)=\sum_{i=0}^{n} x_{i} S\left(x_{i}, a, b\right)=\frac{1}{2} S(1, a, b) .
$$

Поскольку $S(1, a, b)=0$, то $J(1, a, b)=0$.

Таким образом, имеет место (7), т. е. $\{,\}_{n, m}$ - йорданова скобка.

Теорема 5. Пусть $F$ - поле характеристики 0 и $n$ - четное число. Тогда йорданова супералгебра $J\left(\Lambda(n, 1),\{,\}_{n, 1}\right)$ простая. $B$ частности, $J\left(\Lambda(n, 1)_{0}\right.$, $\left.\Lambda(n, 1)_{1},\{,\}_{n, 1}\right)$ является простой супералгеброй. Если $n=2$, то $J\left(\Lambda(n, 1)_{0}\right.$, $\left.\Lambda(n, 1)_{1},\{,\}_{n, 1}\right)$ - исключительная супералгебра.

ДоказАТЕЛЬСтво. Обозначим скобку $\{,\}_{n, 1}$ через $\{$,$\} . По лемме 10$

$$
\begin{gathered}
\left\{x_{i}, x_{j}\right\}=1, i<j, \quad\left\{x_{i}, y_{1}\right\}=1, i, j=0, \ldots, n, \\
D\left(x_{i}\right)=-\sum_{j=0}^{i-1} x_{j}+\sum_{j+1}^{n} x_{j}, i=0, \ldots, n, \quad D\left(y_{1}\right)=-\left(x_{0}+\cdots+x_{n}\right) .
\end{gathered}
$$

Повторяя рассуждения теоремы 4 , можно считать, что алгебра $\Lambda(n, 1)$ содержит такой простой идеал $I$, что $\{I, \Lambda(n, 1)\} \subseteq I$ и $x_{i} \notin I$. 
Поскольку $\Lambda(n, 1)=F\left[x_{0}, \ldots, x_{n-1}, y_{1}\right]+x_{n} F\left[x_{0}, \ldots, x_{n-1}, y_{1}\right]$ и алгебра $\Lambda(n, 1)$ не имеет делителей нуля, то $F\left[x_{0}, \ldots, x_{n-1}, y_{1}\right] \cap I \neq 0$.

Пусть $z(n)=\sum_{i=0}^{n}(-1)^{i} x_{i}$. По теореме 2 , п. 2, для любого $a \in \Lambda(n)$ имеем $\{z(n), a\}=\{z(n), a\}_{n}=0$. Поэтому отображение $\varphi: \Lambda(n, 1) \mapsto \Lambda(n, 1)$, определенное правилом $\varphi(a)=\{z(n), a\}$, является дифференцированием. Кроме того, $\phi(I)=\{z(n), I\} \subseteq I$.

Пусть $f \in F\left[x_{0}, \ldots, x_{n-1}, y_{1}\right] \cap I$ и $f \neq 0$. Тогда

$$
f=f_{0} y_{1}^{k}+f_{1} y_{1}^{k-1}+\cdots+f_{k},
$$

где $f_{i} \in F\left[x_{0}, \ldots, x_{n-1}\right], i=0, \ldots, k$. Предположим, что $f-$ многочлен наименьшей степени $k \geq 1$. Тогда

$$
\phi(f)=\left(k f_{0} y_{1}^{k-1}+(k-1) f_{1} y_{1}^{k-2}+\cdots+f_{k-1}\right) \phi\left(y_{1}\right) \in I,
$$

так как $\phi\left(f_{i}\right)=0, i=0, \ldots, k$. Поскольку $I-$ простой идеал, в силу выбора $f$ получаем $\varphi\left(y_{1}\right) \in I$. Следовательно, $I=\Lambda(n, 1)$, так как $\varphi\left(y_{1}\right)=\left\{z(n), y_{1}\right\}=1$. Поэтому $f \in F\left[x_{0}, \ldots, x_{n-1}\right] \cap I$.

Пусть $f \in F\left[x_{0}, x_{1}, \ldots, x_{n-1}\right] \cap I, f \neq 0$. Тогда $f=f_{k}+f_{k-1}+\cdots+f_{0}$, где каждый ненулевой $f_{i}$ - однородный многочлен из $F\left[x_{0}, x_{1}, \ldots, x_{n-1}\right]$ степени $i$, $i=0, \ldots, k$. Пусть многочлен $f$ имеет наименьшую степень $k$.

Предположим, что $k>0$. Пусть $u=x_{n}-y_{1}$. Тогда $\left\{u, x_{i}\right\}=0, i=$ $0 \ldots, n-1$, и $D(u)=x_{n}$. Повторяя рассуждения теоремы 4 , получаем

$$
\left\{u, x_{0}^{i_{0}} \ldots x_{n-1}^{i_{n-1}}\right\}=-\left(\sum_{j=0}^{n-1} i_{j}-1\right) D(u) x_{0}^{i_{0}} \ldots x_{n-1}^{i_{n-1}}=-\left(\sum_{j=0}^{n-1} i_{j}-1\right) x_{n} x_{0}^{i_{0}} \ldots x_{n-1}^{i_{n-1}} .
$$

Поэтому $\left\{u, f_{i}\right\}=-(i-1) x_{n} f_{i}$, где $i=0, \ldots, k$. Отсюда получаем

$$
\{u, f\}=\left(-(k-1) f_{k}-(k-2) f_{k-1}+\cdots+f_{0}\right) x_{n} .
$$

Поскольку $\{u, f\} \in I, I-$ простой идеал и $x_{n} \notin I$, то

$$
g=-(k-1) f_{k}-(k-2) f_{k-1}+\cdots+f_{0} \in F\left[x_{0}, x_{1}, \ldots, x_{n-1}\right] \cap I .
$$

Тогда $(k-1) f+g \in I$ и степень многочлена $(k-1) f+g$ меньше, чем $k$. В силу выбора $f$ можно считать, что $f$ - однородный многочлен степени $k$.

Повторяя рассуждения теоремы 4, получаем $k=0, f \in F$ и $I=\Lambda(n, 1)$.

Таким образом, супералгебра $J\left(\Lambda(n, 1),\{,\}_{n, 1}\right)$ простая. Тогда по теореме $1 J\left(\Lambda(n, 1)_{0}, \Lambda(n, 1)_{1},\{,\}_{n, 1}\right)$ - простая супералгебра. В силу лемм 8 и 10 $J\left(\Lambda(2,1)_{0}, \Lambda(2,1)_{1},\{,\}_{2,1}\right)$ - исключительная супералгебра.

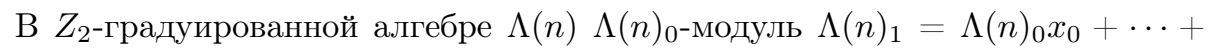
$\Lambda(n)_{0} x_{n}$, т. е. порождается $n+1$ элементами. Возникает вопрос о числе порождающих модуля $\Lambda(n)_{1}$. Здесь имеет место

Теорема 6 [32]. Пусть $R$ - поле действительных чисел,

$$
\Lambda(n)=R\left[x_{0}, \ldots, x_{n}\right] /\left(S^{n}\left(x_{0}, \ldots, x_{n}\right)\right),
$$

$\Lambda(n)_{0}$ - подалгебра в $\Lambda(n)$, порожденная мономами четной степени, $\Lambda(n)_{1}=$ $\Lambda(n)_{0} x_{0}+\cdots+\Lambda(n)_{0} x_{n}$. Тогда $\Lambda(n)_{0}$-модуль $\Lambda(n)_{1}$ не может быть порожден меньшим, чем $n+1$, числом элементов.

Пусть $C$ - поле комплексных чисел и $\Lambda(n)=C\left[x_{0}, \ldots, x_{n}\right] /\left(S^{n}\left(x_{0}, \ldots, x_{n}\right)\right)$. Тогда, как показано в [30], модуль $\Lambda(n)_{1}$ порождается либо $\frac{n+1}{2}$ элементами, 
если $n$ нечетное, либо $\frac{n}{2}+1$ элементами, если $n$ четное. А именно, $u_{k}=x_{2 k}+$ $i x_{2 k+1}, k=0, \ldots, \frac{n+1}{2}-1$, если $n$ нечетное, и $u_{k}=x_{2 k}+i x_{2 k+1}, k=0, \ldots, \frac{n}{2}-1$, $u_{\frac{n}{2}}=x_{n}$, если $n$ четное. Поэтому $\Lambda(n)_{0}$-модуль $\Lambda(n)_{1}$ не может быть порожден меньшим, чем $\left[\frac{n}{2}\right]+1$, числом элементов.

Отсюда следует

Теорема 7. Пусть $F=R(C)$ - поле действительных (комплексных) чисел и $J=A+M-$ йорданова супералгебра. Предположим, что $J=J\left(\Lambda(n)_{0}, \Lambda(n)_{1}\right.$, $\left.\{,\}_{n}\right)$, если $n$ - нечетное число, или $J=J\left(\Lambda(n, 1)_{0}, \Lambda(n, 1)_{1},\{,\}_{n, 1}\right)$, если $n-$ четное число. Тогда $J-$ простая супералгебра. Число порождающих нечетной части $M$ как $A$-модуля не меньше, чем $n+1$, если $F=R$, и не меньше, чем $\left[\frac{n}{2}\right]+1$, если $F=C$. Супералгебра $J\left(\Lambda(2,1)_{0}, \Lambda(2,1)_{1},\{,\}_{2,1}\right)$ исключительная. Более того, если $n>1$ или $F=R$, то супералгебра $J$ не изоморфна супералгебре йордановой скобки.

ДоказАтельство. Докажем последнее утверждение. Пусть $n>1$. Предположим, что супералгебра $J=A+M$ изоморфна супералгебре йордановой скобки $J(\Gamma,\{\}$,$) , где \Gamma=\Gamma_{0}+\Gamma_{1}-$ ассоциативная коммутативная супералгебpa. Тогда $\Gamma_{0}+\Gamma_{1} \xi-$ четная часть, а $\Gamma_{1}+\Gamma_{0} \xi-$ нечетная часть супералгебры $J(\Gamma,\{\}$,$) . Для элемента a \in \Gamma_{1}$ имеем $a \xi \cdot a=-a^{2} \xi=0$. Следовательно, $\left(\Gamma_{0}+\Gamma_{1} \xi\right)$-модуль $\Gamma_{1}+\Gamma_{0} \xi$ имеет кручение. Поскольку $A$-модуль $M$ не имеет кручений, то $\Gamma_{1}=0$. Поэтому нечетная часть $\Gamma_{0} \xi$ супералгебры $J(\Gamma,\{\}$,$) яв-$ ляется однопорожденным $\Gamma_{0}$-модулем. Тогда $A$-модуль $M$ порождается одним элементом; противоречие. Аналогично доказывается случай $F=R$.

Таким образом, $J$ не изоморфна супералгебре йордановой скобки.

Благодарность. Пользуясь случаем, выражаем благодарность В. Ю. Губареву за полезные обсуждения и сделанные замечания. Авторы благодарят рецензента за сделанные замечания.

\section{ЛИТЕРАТУРА}

1. Kantor I. L. Jordan and Lie superalgebras defined by a Poisson algebras // Amer. Math. Soc. 1992. V. 151. P. 55-80.

2. King D., McCrimmon K. The Kantor construction of Jordan superalgebras // Comm. Algebra. 1992. V. 20, N 1. P. 109-126.

3. King D., McCrimmon K. The Kantor doubling process revisited // Comm. Algebra. 1995. V. 23, N 1. P. 357-372.

4. McCrimmon K. Speciality and nonspeciality of two Jordan superalgebras // J. of Algebra. 1992. V. 149 , N 2. P. 326-351.

5. Шестаков И. П. Супералгебры и контрпримеры // Сиб. мат. журн. 1991. Т. 32, № 6. C. $187-196$.

6. Скосырский В. Г. Первичные йордановы алгебры и конструкция Кантора // Алгебра и логика. 1994. Т. 33, № 3. С. 301-316.

7. Шестаков И. П. Квантизация супералгебр Пуассона и специальность йордановых супералгебр пуассонова типа // Алгебра и логика. 1993. Т. 32, № 5. С. 571-584.

8. Шестаков И. П. Квантование алгебр Пуассона и слабая специальность связанных с ними йордановых супералгебр // Докл. АН. 1994. Т. 334, № 1. С. 29-31.

9. Mrtinez C., Shestakov I., Zelmanov E. Jordan superalgebras defined by brackets // J. London Math. Soc. 2001. V. 64, N 2. P. 357-368.

10. Mrtinez C., Zelmanov E. Specializations of Jordan superalgebras // Canad. Math. Bull. 2002. V. 45, N 4. P. 653-671.

11. Shestakov I. On speciality of Jordan brackets // Algebra Discrete Math. 2009. V. 3. P. 94-101.

12. Желябин B. Н., Тихов А. С. Алгебры Новикова - Пуассона и ассоциативные коммутативные дифференциальные алгебры // Алгебра и логика. 2008. Т. 47, № 2. С. 186-202. 
13. Zakharov A. S. Novikov-Poisson algebras and superalgebras of Jordan brackets // Comm. Algebra. 2014. V. 42, N 5. P. 2285-2298.

14. Желябин В. Н., Захаров А. С. Специальность йордановых супералгебр, связанных с алгебрами Новикова - Пуассона // Мат. заметки. 2015. Т. 97, № 3. С. 359-367.

15. Pchelintsev S. V., Shestakov I. P. Prime $(-1,1)$ and Jordan monsters and superalgebras vector type // J. Algebra. 2015. V. 423, N 1. P. 54-86.

16. Пчелинцев C. В., Шашков О. В. Простые конечномерные правоальтернативные супералгебры абелева типа характеристики нуль // Изв. РАН. Сер. мат. 2015. Т. 79, № 3. C. $131-158$.

17. Желябин В. Н. Простые специальные йордановы супералгебры с ассоциативной нильполупростой четной частью // Алгебра и логика. 2002. Т. 41, № 3. С. 276-310.

18. Желябин В. Н., Шестаков И. П. Простые специальные супералгебры с ассоциативной четной частью // Сиб. мат. журн. 2004. Т. 45, № 5. С. 1046-1072.

19. ЖКелябин В. Н. Дифференциальные алгебры и простые йордановы супералгебры // Мат. тр. 2009. Т. 12, № 2. С. 41-51.

20. Zhelyabin V. N. Differential algebras and simple Jordan superalgebras // Sib. Adv. Math. 2010. V. 20, N 3. P. 223-230.

21. Желябин В. Н. Новые примеры простых йордановых супералгебр над произвольным полем характеристики нуль // Алгебра и анализ. 2012. Т. 24, № 4. С. 84-96.

22. Желябин B. Н. Простые йордановы супералгебры с ассоциативной ниль-полупростой четной частью // Сиб. мат. журн. 2016. Т. 57, № 6. С. 1262-1279.

23. ЖКелябин В. Н. Вложение йордановых супералгебр в супералгебры йордановых скобок // Сиб. мат. журн. 2020. Т. 61, № 1. С. 78-95.

24. Желябин В. Н. Примеры первичных йордановых супералгебр векторного типа и супералгебр типа Ченга - Каца // Сиб. мат. журн. 2013. Т. 54, № 1. С. 49-56.

25. Шестаков И. П. Простые $(-1,1)$-супералгебры // Алгебра и логика. 1998. Т. 37, № 6. C. $721-739$.

26. Billiga $Y$., Futorny $V$. Lie algebras of vector fields on smooth affine varieties // Comm. Algebra. 2018. V. 46, N 8. P. 3413-3429.

27. Jordan D. On the ideals of a Lie algebra of derivations // J. London Math. Soc. 1986. V. 33. P. 33-39.

28. Siebert $T$. Lie algebras of derivations and affine algebraic geometry over fields of characteristic 0 // Math. Ann. 1996. V. 305. P. 271-286.

29. Cantarini N., Kac V. G. Classification of linearly compact simple Jordan and generalized Poisson superalgebras // J. of Algebra. 2007. V. 313, N 1. P. 100-124.

30. Желябин В. Н. Дополнение к теореме Блока и к теореме Попова о дифференциально простых алгебрах // Сиб. электрон. мат. изв. 2019. Т. 16. С. 1375-1384.

31. Posner E. C. Differentiable simple rings // Proc. Amer. Math. Soc. 1960. V. 11, N 3. P. 337-343.

32. Swan R. G. Vector bundles and projective modules // Trans. Amer. Math. Soc. 1962. V. 105, N 2. P. 264-277.

Поступила в редакиию 16 декабря 2019 г.

После доработки 20 марта 2020 г.

Принята к публикации 17 июня 2020 г.

Желябин Виктор Николаевич

Институт математики им. С. Л. Соболева СО РАН, пр. Академика Коптюга, 4, Новосибирск 630090

vicnic@math.nsc.ru

Захаров Антон Станиславович

Новосибирский государственный университет,

ул. Пирогова, 1, Новосибирск 630090;

Новосибирский государственный технический университет, пр. К. Маркса 20, Новосибирск 630073

antzakh@gmail.com 